\title{
Optical Spectroscopy of Quantum Dots in High Magnetic Fields
}

\author{
A. BABIŃSKI \\ Institute of Experimental Physics, Warsaw University \\ Hoża 69, 00-681 Warszawa, Poland
}

\begin{abstract}
Optical spectroscopy measurements of single InAs/GaAs self-assembled quantum dots have been performed. The multiexcitonic emission from the $s^{-}, p$, and $d$-shells of a single dot is observed and investigated in magnetic field up to $28 \mathrm{~T}$. The Zeeman splitting of the $s$-shell excitons has been found to depend on the dot morphology. While the energy-splitting in flat (2 nm height) dots linearly increases with magnetic field, its significant non-linearity is observed for larger in size, tall (4 $\mathrm{nm}$ height) dots. The effect of magnetic field on the orbital motion of carriers in dots has also been investigated. It has been found that the modified Fock-Darwin pattern explains the observed evolution of the emission from highly-excited single tall quantum dot.
\end{abstract}

PACS numbers: 78.67.Hc, 78.55.Cr, 75.75.+a

\section{Introduction}

Semiconductor quantum dots (QDs) offer a unique opportunity to study electron-electron interactions in strongly confined systems [1]. Significant interest of a scientific community in studies of QDs is driven by both their fundamental properties and by their possible applications in quantum information technology. Of prime interest are the properties of excitons confined in QDs, which are being thoroughly investigated over last 10 years. Much less experimental work has been devoted to highly-excited QDs, in which optical recombination due to multiexcitonic configurations can be studied. The properties of those configurations, and in particular their evolution in magnetic field can provide important information, e.g. on the lateral potential localizing electrons and holes in QDs.

The "analytical" example of a semiconductor dot is a laterally confined two-dimensional (2D) system, with a harmonic lateral confinement potential. The discrete and energetically equidistant single-particle levels of such a dot are expected as a simple solution of the $2 \mathrm{D}$ harmonic oscillator. These levels are referred to 
as $s, p, d$, (atomic-like) shells with their two-, four-, six-fold, ... degeneracy, respectively. In a photoluminescence (PL) experiment these electron and hole shells are filled with carriers and the emission due to their recombination is observed. As the Pauli principle forbids occupation of the energy levels with more than two carriers (of opposite spins), higher-energy shells become occupied with increasing excitation power. Dipole-allowed recombination of electrons and holes from the respective shells of the same quantum numbers can be observed in PL spectra. This shell-filling is a characteristic feature of a three-dimensional confinement and it has been confirmed in a series of experiments [2, 3]. In order to observe the pattern in PL experiments from large numbers of dots, low inhomogeneous broadening of the shell-related peaks is required, which can be obtained by proper growth procedures and a post-growth thermal annealing $[4,5]$.

This simple model of electron-hole recombination must be modified as carriers in QDs are strongly localized. Due to electron-electron interaction, the picture of many-excitonic droplets is more relevant [6, 7]. Some effects related to the interaction, as e.g. an apparent band-gap renormalization of the $s$-shell emission with increasing excitation power, can be observed in the spectra from large numbers of dots. However most details of the effects are obscured by inhomogeneous broadening of emission from large arrays of QDs. A single-dot spectroscopy is needed to overcome the problems. Such experiments mostly deal with the emission from the lowest $s$-shell of QDs [8], although the shell structure of a highly-excited single QD has also been investigated [9-11]. However, up to now, no measurement of magnetic-field evolution of the emission from a single highly-excited QD has been reported. The magnetospectroscopic study of highly-excited single QDs is presented in this paper.

\section{Experimental setup}

An experimental setup for the single-dot spectroscopy in high magnetic fields has been made operational in Grenoble High Magnetic Field Laboratory. A substantial size of Bitter magnets providing high magnetic fields imposes the use of fibre optics in such experiments. Fibre optics enables a precise access to the investigated sample and reduces mechanical effects, which may move the sample with respect to the optical setup. High stability of the setup is of a prime importance for optical measurements of single dots. Such stability has been assured in the setup described in this section [12]. The main part of the optical setup is shown in Fig. 1. The laser excitation ( $\mathrm{Ar}^{+}$laser or Ti-sapphire tuneable laser) is led to the sample via a single-mode fibre and focussed by a combination of two aspheric micro-lenses. The obtained spot size is of the order of $10 \mu \mathrm{m}$. The emission signal is collected by a $600 \mu \mathrm{m}$ core multi-mode fibre, analyzed by a $1 \mathrm{~m}$ double grating monochromator and detected by a liquid nitrogen cooled CCD-camera.

Single QDs were geometrically isolated from the sample by the formation of submicron-size mesas. To address specific positions on the sample, it was mounted 


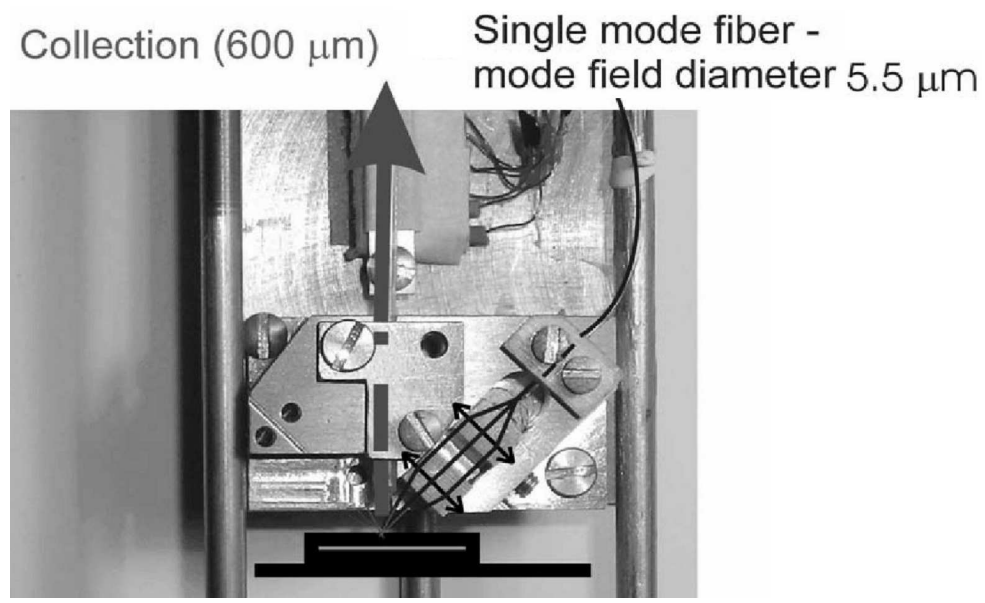

Fig. 1. Optical setup for single-dot measurements in high magnetic fields.

on piezo-driven stages. Mesas of a different size form an array, which makes navigation over the sample more easy. The $z$-axis stage also allows the focusing of excitation light. It has been found that the stable position of the sample below the spatially fixed fibre optics has not been compromised during experiments in magnetic fields up to $28 \mathrm{~T}$. The setup is placed in the liquid helium insert of a cryostat at $T=4.2 \mathrm{~K}$ and the cryostat may be located in a Bitter-magnet supplying magnetic field up to $28 \mathrm{~T}$.

\section{Samples}

Samples investigated in this work were fabricated using molecular beam epitaxy in the Institute for Microstructural Sciences, NRC, Canada. The samples were grown on an $n^{+}$-GaAs substrate and contain a single layer of QDs. Nominally InAs/GaAs QDs were formed by self-assembly on a $600 \mathrm{~nm}$ undoped GaAs buffer layer and capped with a $100 \mathrm{~nm}$ GaAs layer. An In-flush growth procedure was used [13] which starts with covering the deposited dots with a protection layer of a few nm thickness. Then the growth is stopped and the exposed tips of the dots are depleted of In atoms and subsequently covered with GaAs. Resulting dot geometry can be approximately described by a disk of a well-defined thickness, which improves significantly the inhomogeneous distribution of dot's sizes. Two kinds of structures were grown: in the tall (flat) dots the thickness of the protection layer was 5 (3) nm, resulting in dots with a height of about 4 (2) nm after the flush. The sample with tall dots has been additionally annealed after the growth $\left(30 \mathrm{~s}\right.$ at $\left.850^{\circ} \mathrm{C}\right)$ to shift the emission into the sensitivity range of a CCD-camera and to decrease the confining potential in the QD.

The quality of the investigated samples was confirmed by measurements on large numbers of dots. The power dependence of the PL from tall dots in a $20 \mu \mathrm{m}$ size mesa can be appreciated in Fig. 2. When excited with low power, the spectrum 
consists of a series of sharp peaks mainly due to emission from the $s$-shell of QDs. With increasing power the emission from the higher-energy shells (subsequently: $p, d$, and $f$ ) starts to be visible. The change in the line shape of the spectrum as a function of excitation power can be noticed. While its fine structure can be observed when excited with low power, with increased power the spectrum becomes featureless. This results from a large number of possible multiexcitonic configurations of each dot of the array and possible interactions with continuum of states in the wetting layer $[14,15]$.

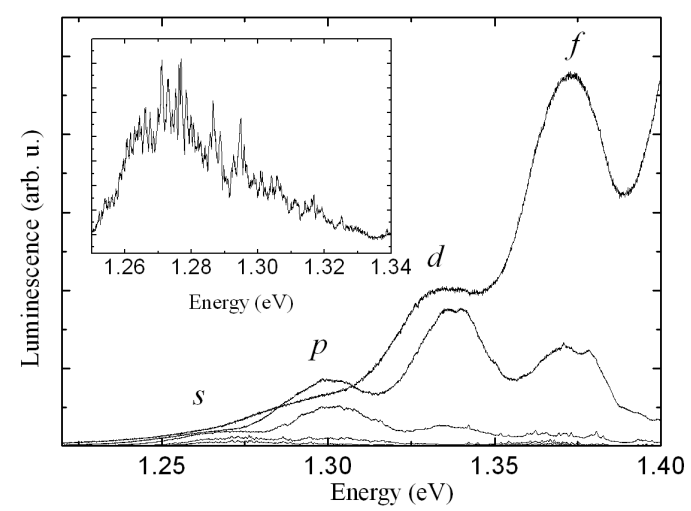

Fig. 2. The power dependence of a luminescence from a large mesa with tall dots $(T=4.2 \mathrm{~K}, B=0 \mathrm{~T})$. The spectrum excited with low power is shown in the inset. The filling of subsequent shells can be noticed.

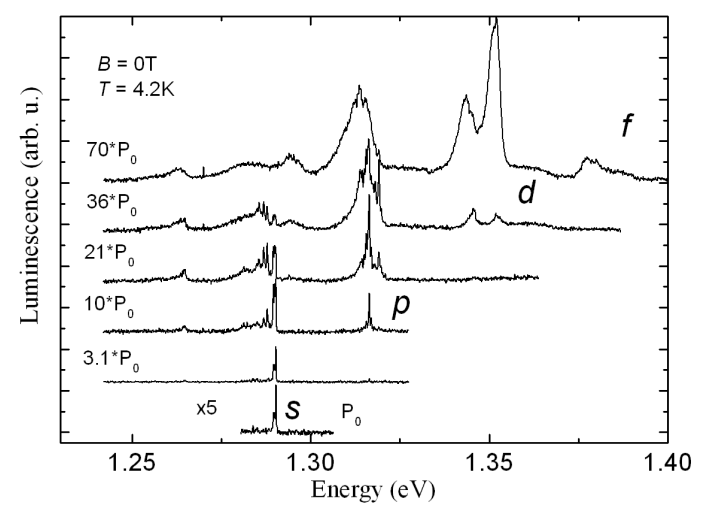

Fig. 3. The power dependence of a luminescence from a single tall InAs/GaAs dot $\left(P_{0} \approx 1 \mathrm{~W} / \mathrm{cm}^{2}\right)$. The filling of subsequent shells can be noticed. Weak emission feature $25 \mathrm{meV}$ below the $s$-shell emission is related to a phonon replica of the emission.

This effect can be better seen in the spectra from a single tall QD (see Fig. 3). At low excitation power density a few emission lines can be observed, 
which are related to excitonic recombination from the $s$-shell. Depending on the dot investigated, the low-power spectrum may consist of a single line, accompanied by several weak features or of a series of lines with a characteristic pattern $[16,17]$. The multiline spectrum has been attributed to different charge states of a single dot. With increasing excitation power an additional emission from the $p$-shell in form of sharp emission lines emerges, as well as new features from the $s$-shell can be seen. Clear splitting of the $p$-shell emission can be appreciated in spectra obtained with increased excitation. Further increase of excitation power results in the emission from the $d$-shell and substantial broadening of the emission from the $s$ - and $p$-shells. As opposite to the emission from lower-energy shells - no fine structure of the $d$-shell emission can be observed. Finally, when the emission from the $f$-shell starts to be visible, the emission from lower-lying shells becomes featureless.

\section{Quantum dots in high magnetic field}

The quantum confinement in QDs can be effectively modified by the application of a magnetic field. The magnetic field applied perpendicularly to the initial 2D plane lifts the orbital degeneracy of a single harmonic oscillator. The resulting Fock-Darwin (FD) diagram [18] displays a characteristic diamond-like level crossing pattern, which implies the reconstruction of the 2D Landau-level structure in the high field limit (see Fig. 4). The energy $E_{n l}$ of an electronic (hole) state with a radial quantum number $n(=0,1,2, \ldots)$, and an angular quantum number $l(=0, \pm 1, \pm 2)$ confined in a parabolic potential $\hbar \varpi_{0}^{\beta}$ in the presence of a perpendicular magnetic field $B$ is given by: $E_{n l}^{\beta}=(2 n+|l|+1) \hbar \Omega_{\beta}+\frac{1}{2} l \hbar \varpi_{\mathrm{c}}^{\beta}$ with $\varpi_{\mathrm{c}}^{\beta}=e B / m_{\beta}$ and $\Omega_{\beta}=\sqrt{\left(\varpi_{0}^{\beta}\right)^{2}+\frac{1}{4}\left(\varpi_{\mathrm{c}}^{\beta}\right)^{2}}$, where $\beta$ stands for electron or hole. As it can be seen in Fig. 4 the $s$-shell — a single particle $(0,0)$ level — shifts diamagnetically in magnetic field. The magnetic field removes the degeneracy of the $p$-shell — single particle $(0,1)$ and $(0,-1)$ levels split. The energy splitting

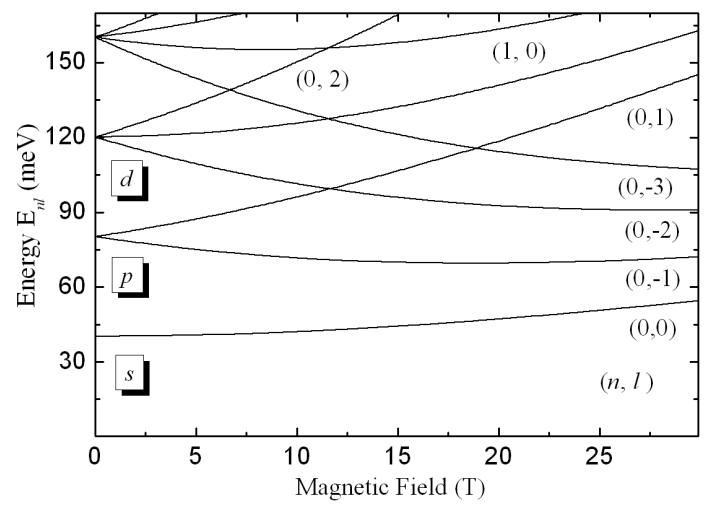

Fig. 4. Calculated single-particle states versus magnetic field for a parabolic potential with $\hbar \varpi_{0}^{\mathrm{c}}=40 \mathrm{meV}$. 
equals electron (hole) cyclotron energy. Similarly the degeneracy of the $d$-shell: $(0,2)$ and $(0,-2)$ levels - is also removed by magnetic field*. The FD pattern can be observed in low-temperature magneto-tunneling experiments on relatively large dots, electrostatically shaped from the 2D electron gas [19-22]. The electronic FD spectrum has also been observed in capacitance measurements on self-assembled QDs [23].

In optical experiments on self-assembled QDs, this picture becomes more complicated as both electrons and holes are involved in the transitions and interactions between the carriers cannot be neglected. Until now, the magneto-PL from the excited $p, d, \ldots$, shells has only been investigated on ensembles of QDs: induced by self-organized InP islands on InGaAs/GaAs [24] or self-assembled [25-28]. The FD pattern was observed in those experiments with some discrepancies related to the asymmetry of investigated dots [24]. Moreover, although obscured by inhomogeneous broadening of observed emission lines, some effects of electron-electron interaction at the field-induced crossings of single-particle levels have also been observed [26]. To uncover those details, the single-dot spectroscopy has been applied in high magnetic fields using the setup described in Sect. 2 of this paper.

\subsection{Zeeman effect}

Let us consider first the Zeeman interaction of magnetic field with spins of carriers confined in QDs [29, 30]. It is usually assumed that excitons in InAs/GaAs QDs are composed of electrons and heavy holes. This is because substantial strain in the dots splits the heavy and light holes in energy by at least several tens on meV. Four excitons can be formed from heavy holes of spin $J_{\mathrm{h}}=3 / 2$, and its projection along the growth direction $J_{\mathrm{h}, z}= \pm 3 / 2$ and an electron of spin $S=1 / 2$, and $S_{\mathrm{e}, z}= \pm 1 / 2$. The excitons are characterized by angular momenta $M=S_{\mathrm{e}, z}+J_{\mathrm{h}, z}[30]$. The states with $|M|=2$ are optically inactive (dark excitons) and the states with $|M|=1$ are optically active (bright excitons). Both states are split due to a short-range electron-hole exchange interaction. The bright excitons, which are degenerate in a symmetric lateral potential, split in asymmetric potential due to the long-range electron-hole exchange interaction. In high magnetic fields, in which the effect of the exchange splitting can be neglected, the energy splitting of two components of the neutral exciton may be therefore expressed as $\Delta E=g^{*} \mu_{\mathrm{B}} B=\left|g_{\mathrm{e}}+g_{\mathrm{h}}\right| \mu_{\mathrm{B}} B$. The same splitting can be expected from the emission from a (negatively) charged exciton, as it results from the Zeeman hole splitting in the initial state (two electrons have opposite spins) and the Zeeman electron splitting in the final state. Equal values of effective $g^{*}$ factors for neutral charged (and doubly charged) excitons in single QDs have also been confirmed experimentally [31]. If a band-mixing is excluded, constant values of $g_{\mathrm{e}}$ and $g_{\mathrm{h}}$ can be used over a broad range of magnetic fields [30]. In the case of flat InAs/GaAs dots, this picture of a constant excitonic $g^{*}$ factor also holds in an extended range

\footnotetext{
${ }^{*}$ It must be noted that the $(1,1)$ level, which is sometimes referred to as the $d_{0}$ subshell is degenerate with the $d_{+}$and $d_{-}$subshells only in parabolic potential.
} 
of magnetic fields [16]. As it can be seen in Fig. 5 the excitonic emission line splits in magnetic field and the centre of gravity of the split doublet shifts diamagnetically towards higher energies. The splitting depends linearly on magnetic field, with a small discrepancy in the highest field range (see Fig. 6).

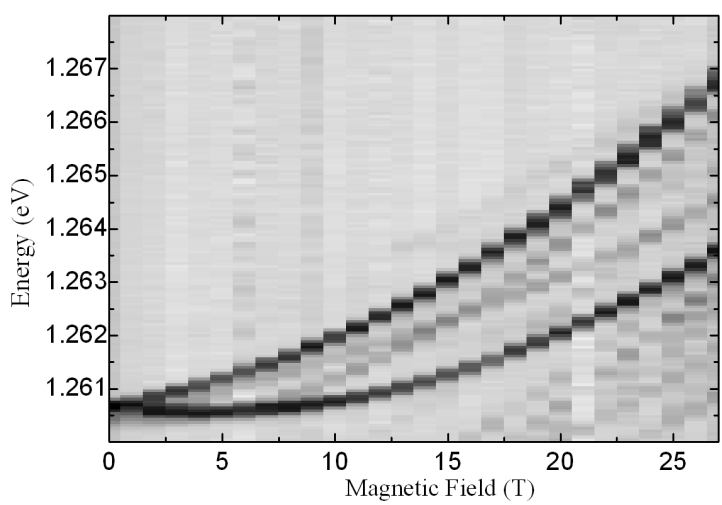

Fig. 5. The surface plot of normalized emission from a single flat InAs/GaAs QD in magnetic field up to $27 \mathrm{~T}$. The Zeeman splitting and the diamagnetic shift can easily be observed.

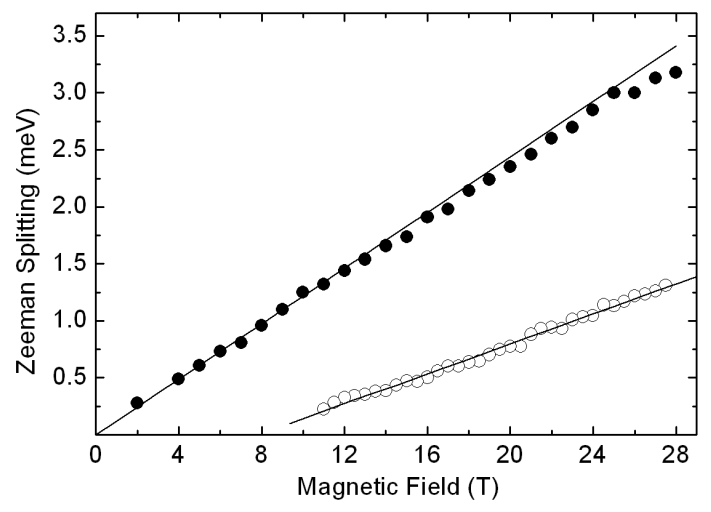

Fig. 6. The energy splitting of excitonic emission lines from flat (closed symbols) and tall (open symbols) InAs/GaAs quantum dots in magnetic field up to $27 \mathrm{~T}$. Continuous lines present the linear fits to experimental data.

The experimental picture changes dramatically when tall dots are investigated. It has been found that the excitonic emission lines from those QDs do not split in magnetic field lower than 10...14 T (see Fig. 7). The energy splitting observed in higher magnetic fields is a linear function of the field, however the effective excitonic $g^{*}$ factor deduced from the dependence is considerably smaller than in the case of flat dots (see Fig. 6). It must be noted that the non-linearity, 


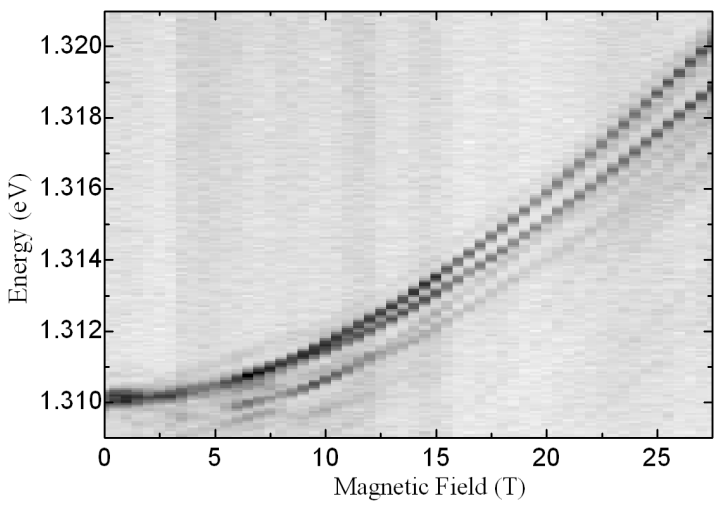

Fig. 7. The surface plot of normalized emission from a single tall InAs/GaAs in magnetic field up to $27 \mathrm{~T}$.

although often present in quantum wells, has not been previously observed in QDs. A similar effect of the heavy hole - light hole mixing [32] has also been proposed to explain the observed behaviour [16]. In fact the effect of mixing, although very weak, must be considered in QDs in order to explain the exchange-interaction splitting of a bright exciton in zero-magnetic field $[33,34]$. The experimental data suggest that in tall dots the effect must be much stronger, which presumably leads to the apparent non-linearities of the splitting.

\subsection{Orbital effects in magnetic field}

If the condition of a "hidden symmetry": $m_{\mathrm{e}} \varpi_{0}^{\mathrm{e}}=m_{\mathrm{h}} \varpi_{0}^{\mathrm{h}}$ is fulfilled [7], which implies an identical form of electronic and hole wave functions on their corresponding $s, p, d, \ldots$, shells, the energy difference between the electronic and hole single-particle levels of the same sets of quantum numbers $\Delta E_{n l}$ (which are involved in dipole-allowed optical transitions) can be expressed in terms of an "excitonic" (electron-hole pair) FD diagram. The energy $\Delta E_{n l}=E_{\mathrm{g}}+(2 n+$ $|l|+1) \hbar \Omega+\frac{1}{2} l \hbar \varpi_{\mathrm{c}}$ with $\varpi_{0}=\varpi_{0}^{\mathrm{e}}+\varpi_{0}^{\mathrm{h}}$ and the cyclotron frequency $\varpi_{\mathrm{c}}=e B / \mu^{*}=$ $\left(1 / m_{\mathrm{e}}+1 / m_{\mathrm{h}}\right) e B$. The effective energy gap $E_{\mathrm{g}}$ in this model accounts for the energy difference between the top of the valence band and the bottom of the conduction band.

To examine the validity of the "excitonic" FD model, the magneto-spectroscopic measurements of a highly excited single QD have been performed. The emission (see Fig. 8) consists of a series of lines due to recombination of excitons from the $s$-shell (at $1.285 \mathrm{eV}$ ) as well as emission lines related to the $p$-shell (at $1.315 \mathrm{eV}$ ). The multiline pattern of the $s$-shell emission results from several charged states probed during the collection of spectra. The $p$-shell related emission lines result from the QD's occupation with more than 3 excitons [9]. The evolution of the spectrum in magnetic field can be appreciated in Fig. 8. The $s$-shell emission lines shift diamagnetically in magnetic field. No Zeeman splitting of these lines can be noticed, which agrees with previously analyzed data. Two 


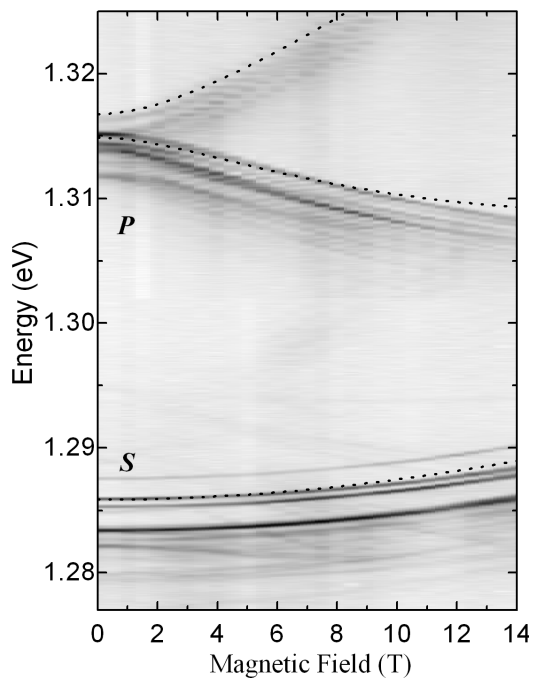

Fig. 8. The surface plot of the emission from a single tall InAs/GaAs in magnetic field up to $14 \mathrm{~T}$. The multiline emission from the $s$-shell, as well as the emission from the $p$-shell can be seen.

groups of the $p$-shell related emission lines can be distinguished in the spectrum, either red-shift or blue-shift in magnetic field. This dependence suggests their attribution to the single particle $p$-shell states with electronic angular momenta $l=-1$ and $l=+1$ (referred to as the $p_{-}$and $p_{+}$levels, respectively). In the FD model of a symmetric lateral potential, these single particle states are degenerate in zero magnetic field. However the electron-electron interactions of multiexcitonic configurations [7] and a possible asymmetry of the lateral potential [35] mix the $p_{-}$and $p_{+}$-related states in zero magnetic field. Resulting are the $p_{x}$ and $p_{y}$ levels, which in high magnetic field evolve into the $p_{-}$and $p_{+}$levels. Using a perturbation theory, one can describe the energy difference between the emission lines related to the $p_{x}$ and $p_{y}$ levels by the equation: $\Delta E^{\prime}(B)=E_{\Delta}+\sqrt{\delta^{2}+\left(\hbar \varpi_{\mathrm{c}}\right)^{2}}$. The energy splitting $\Delta E^{\prime}(B)$ accounts for possible effects of electron-electron interactions and the zero-field splitting of the single-particle $p$ levels due to asymmetry of the lateral confining potential. It has been found that the splitting can be reproduced with a single reduced electron-hole effective mass $\mu^{*}=0.057 \pm 0.0005 m_{0}$, the zerofield splitting $\delta=5.5 \pm 0.5 \mathrm{meV}$, and the energy $E_{\Delta}$ ranging from $-4.35 \mathrm{meV}$ to $-2.6 \mathrm{meV}[36]$. The magnitude of the zero-field splitting agrees with the values of electronic $p$-shell splitting found in far-infrared experiments, being of the order of $5 \mathrm{meV}[37,38]$ and a theoretical value $(3 \mathrm{meV})$ for a similar lens-shaped dot [35]. Using the obtained values of reduced mass $\mu^{*}$ and the zero-field splitting $\delta$, as well as the confinement energy $\hbar \omega_{0}=31.3 \mathrm{meV}$ one can describe the magnetic-field evolution of the $p_{x}$-shell-related emission lines (see dotted line in Fig. 8) (the high-field discrepancies must result from the changes of excitonic binding energy in 
magnetic field, not accounted for in this model). Similar approach can be applied to describe the field-dependence of the $p_{y}$-related emission lines and in particular its apparent interaction with the $d_{-}$-related emission lines (not shown in Fig. 8), which occurs at higher magnetic field [26]. It must also be noted that within good approximation, in order to follow different emission lines, different, although constant values of interaction energy must be added to the single-particle FD pattern. This reminds the constant-interaction approach, which is used to describe electronic states in electrostatically-defined QDs [20].

As it has been shown, our experimental data can be well described by the FD model modified by phenomenological parameters reflecting electron-electron interactions and a possible asymmetry of localizing potential. This suggests that electron-electron interactions in the investigated dot can be treated as a perturbation to the single-particle shell structure. It must be noted however that this picture may not be valid in description of all types of QDs. In particular in smaller InAs/GaAs dots of larger localization energy, a complicated pattern of excitonic polaron states has been observed in PL excitation spectra, which results from a strong coupling between the mixed exciton-longitudinal optical (LO) phonon states [39]. Therefore, it seems obvious that more experimental work is necessary to explain the behaviour of multi-excitonic configurations in QDs of different morphology, and in particular its evolution in magnetic fields. The task can be accomplished using the single-dot setup described in this paper.

\section{Conclusions}

The results of spectroscopic studies of InAs/GaAs QDs in high magnetic fields have been presented. The emission from single QDs has been identified and investigated. The non-linear dependence of the Zeeman splitting on magnetic field of excitonic energy has been observed in relatively large tall dots, which has been attributed to the valence band mixing. The magnetic-field evolution of emission lines due to multiexcitonic configurations of a single QD has also been investigated. Electron-electron interactions in combination with possible effects of asymmetric confinement potential are observed to lift the degeneracy of single-particle states at zero magnetic fields. It has been shown that a modified single-particle approach well describes the experimental data.

\section{Acknowledgments}

The work presented in this paper results from a collaboration between Grenoble High Magnetic Field Laboratory, CNRS, Grenoble, France, where the experiments in high magnetic field have been performed, Institute for Microstructural Sciences (IMS), NRC, Canada, where samples investigated were grown, prepared, and where initial measurements took place, and Universität Dortmund, Experimentelle Physik IIa, Dortmund, Germany. The author expresses his gratitude to M. Potemski, who supervised experimental work done in Grenoble, S. Raymond, G. Ortner, M. Vachon, and S. Awirothananon, who took part in measurements 
and Z. Wasilewski and J. Lapointe, who respectively grew the samples and prepared them for measurements. Valuable discussion with M. Bayer, W. Sheng, M. Korkusiński, and P. Hawrylak are also acknowledged.

The access to the experimental facility has been supported by the 1 P03B 01429 project of the Ministry of Science and Higher Education and the RITA-CT-2003-505474 EC project. Financial support from EC through the INCO Training and Excellence Fellowship ICA1-2002-70009 and ICA1-2002-70010 is kindly acknowledged.

\section{References}

[1] D. Bimberg, M. Grundmann, N.N. Ledentsov, Quantum Dot Heterostructures, Wiley, New York 1998; L. Jacak, P. Hawrylak, A. Wojs, Quantum Dots, Springer, Berlin 1998.

[2] S. Grosse, J.H.H. Sandmann, G. von Plessen, J. Feldmann, H. Lipsanen, M. Sopanen, J. Tulkki, J. Ahopelto, Phys. Rev. B 55, 4473 (1997).

[3] S. Fafard, Z.R. Wasilewski, C.N. Allen, D. Picard, M. Spanner, J.P. McCaffrey, P.G. Piva, Phys. Rev. B 59, 15368 (1999).

[4] S. Fafard, C.N. Allen, Appl. Phys. Lett. 75, 2374 (1999).

[5] N. Perret, D. Morris, L. Franchomme-Fossé, R. Côté, S. Fafard, V. Aimez, J. Beauvais, Phys. Rev. B 62, 5092 (2000).

[6] A. Wojs, P. Hawrylak, Phys. Rev. B 55, 13066 (1997).

[7] P. Hawrylak, Phys. Rev B 60, 5597 (1999).

[8] see e.g. Optics of Quantum Dots and Wires, Eds. G.W. Bryant, G.S. Solomon, Artech House Boston, London 2005.

[9] M. Bayer, O. Stern, P. Hawrylak, S. Fafard, A. Forchel, Nature 405, 923 (2000).

[10] K. Kheng, L. Besombes, H. Mariette, Physica E 26, 262 (2005).

[11] D. Chithrani, R.L. Williams, J. Lefebvre, P.J. Poole, G.C. Aers, Appl. Phys. Lett. 84, 978 (2005).

[12] A. Babinski, S. Awirothananon, J. Lapointe, Z. Wasilewski, S. Raymond, M. Potemski, Physica E 26, 190 (2005).

[13] Z.R. Wasilewski, S. Fafard, J. McCaffrey, J. Cryst. Growth 201, 1131 (1999).

[14] R.J. Warburton, C. Schäflein, D. Haft, F. Bickel, A. Lorke, K. Karrai, J.M. Garcia, W. Schoenfeld, P.M. Petroff, Nature 405, 926 (2000).

[15] E. Dekel, D. Gershoni, E. Ehrenfreund, D. Spektor, J.M. Garcia, P.M. Petroff, Phys. Rev. Lett. 80, 4991 (1998).

[16] A. Babiński, G. Ortner, S. Raymond, M. Potemski, M. Bayer, W. Sheng, P. Hawrylak, Z. Wasilewski, S. Fafard, A. Forchel, Phys. Rev. B 74, 075310 (2006).

[17] A. Babinski, M. Potemski, S. Raymond, J. Lapointe, Z.R. Wasilewski, accepted by Phys. Status Solidi $C$.

[18] V. Fock, Z. Phys. 47, 446 (1928); C.G. Darwin, Proc. Cambridge Philos. Soc. 27, 86 (1930).

[19] S. Tarucha, D.G. Austing, T. Honda, R.J. van der Hage, L.P. Kouwenhoven, Phys. Rev. Lett. 77, 3613 (1996). 
[20] L.P. Kouwenhoven, D.G. Austing, S. Tarucha, Rep. Prog. Phys. 64, 701 (2001).

[21] M. Ciorga, A. Wensauer, M. Pioro-Ladriere, M. Korkusinski, J. Kyriakidis, A.S. Sachrajda, P. Hawrylak, Phys. Rev. Lett. 88, 256804 (2002).

[22] Electron Transport in Quantum Dots, Ed. J.P. Bird, Kluwer Academic Publishers, Boston 2003.

[23] B.T. Miller, W. Hansen, S. Manus, R.J. Luyken, A. Lorke, J.P. Kotthaus, S. Huant, G. Medeiros-Ribeiro, P.M. Petroff, Phys. Rev. B 56, 6764 (1997).

[24] R. Rinaldi, P.V. Giugno, R. Cingolani, H. Lipsanen, M. Sopanen, J. Tulkki, Phys. Rev. Lett. 77, 342 (1996).

[25] P.P. Paskov, P.O. Holtz, B. Monemar, J.M. Garcia, W.V. Schoenfeld, P.M. Petroff, Phys. Rev. B 62, 7344 (2000).

[26] S. Raymond, S. Studenikin, A. Sachrajda, Z. Wasilewski, S.J. Cheng, W. Sheng, P. Hawrylak, A. Babiński, M. Potemski, G. Ortner, M. Bayer, Phys. Rev. Lett. 92, 187402 (2004).

[27] D. Smirnov, S. Raymond, S. Studenikin, A. Babiński, J. Leotin, P. Frings, M. Potemski, A. Sachrajda, Physica B 346-347, 432 (2004).

[28] S. Awirothananon, W.D. Sheng, A. Babiński, S. Studenikin, S. Raymond, A. Sachrajda, M. Potemski, S. Fafard, G. Ortner, M. Bayer, Jpn. J. Appl. Phys. 43, 2088 (2004).

[29] H.W. van Kesteren, E.C. Cosman, W.A.J.A. van der Poel, C.T. Foxon, Phys. Rev. B 41, 5283 (1990).

[30] M. Bayer, G. Ortner, O. Stern, A. Kuther, A.A. Gorbunov, A. Forchel, P. Hawrylak, S. Fafard, K. Hinzer, T.L. Reinecke, S.N. Walck, J.P. Reithmaier, F. Klopf, F. Schäfer, Phys. Rev. B 65, 195315 (2002).

[31] C. Schulhauser, R.J. Warburton, A. Hoegele, A.O. Govorov, K. Karrai, J.M. Garcia, B.D. Gerardot, P.M. Petroff, Physica E 21, 184 (2004).

[32] G.E.W. Bauer, T. Ando, Phys. Rev. B 37, 3130 (1988).

[33] L. Besombes, K. Kheng, D. Martrou, Phys. Rev. Lett. 85, 425 (2000).

[34] A.V. Koudinov, I.A. Akimov, Yu.G. Kusrayev, F. Henneberger, Phys. Rev. B 70, 241305(R) (2004).

[35] G. Bester, A. Zunger, Phys. Rev. B 71, 045318 (2005).

[36] A. Babinski, M. Potemski, S. Raymond, J. Lapointe, Z.R. Wasilewski, to be published in Phys. Rev. B.

[37] E.A. Zibik, L.R. Wilson, R.P. Green, G. Bastard, R. Ferreira, P.J. Phillips, D.A. Carder, J.-P.R. Wells, J.W. Cockburn, M.S. Skolnick, M.J. Steer, M. Hopkinson, Phys. Rev. B 70, 161305(R) (2004).

[38] S. Hameau, Y. Guldner, O. Verzelen, R. Ferreira, G. Bastard, J. Zeman, A. Lamaitre, J.M. Gérard, Phys. Rev. Lett. 83, 4152 (1999).

[39] V. Preisler, T. Grange, R. Ferreira, L.A. de Vaulchier, Y. Guldner, F.J. Teran, M. Potemski, A. Lemaître, Phys. Rev. B 73, 075320 (2006). 